\title{
Propagación vegetativa de Lisianthus (Eustoma grandiflorum RAF) cv. Abc 2-3 Blue Rim
}

\author{
Vegetative propagation of (Eustoma grandiflorum RAF) cv. Abc 2-3 Blue Rim \\ Eugenio M. Sotomayor León ${ }^{1 *}$, Carla A. Rosas Guerra y Pilar Mazuela ${ }^{1}$
}

\begin{abstract}
RESUMEN
El creciente interés por el cultivo de Lisianthus y la falta de datos experimentales disponibles demanda el estudio del comportamiento fenológico y productivo de esta especie. Uno de los principales aspectos que preocupa al productor de flores es el manejo agronómico y la obtención de plantas de este cultivo. El objetivo de este trabajo fue establecer dos experimentos para evaluar la técnica de propagación y su efecto en el enraizamiento de esquejes bajo distintas concentraciones de auxinas. El material vegetal fue extraído de plantas madre una vez realizado el raleo y pinzamiento de las varas para dejar las más vigorosas. En el primer experimento se utilizó una mesa cerrada (MC), cubierta con polietileno transparente, regada en sustrato hasta saturación. En el segundo experimento se utilizó una mesa bajo nebulización (MBN) con boquillas atomizadoras para el riego. El diseño fue de bloques completos al azar y para separación de medias se usó el test de Duncan. Los tratamientos fueron según concentración de ácido indolbutírico (AIB): T0, testigo, sin AIB; T1, 250 ppm AIB; T2, 500 ppm AIB y T3, 1.000 ppm AIB. Los resultados indican que en el experimento en MC la aplicación de auxina mejora el enraizamiento de esquejes, sin que se observe diferencia significativa entre tratamientos. En el segundo experimento MBN, se observa que en la aplicación de AIB mejora la propagación, obteniéndose mejores resultados con la mayor concentración de auxina.
\end{abstract}

Palabras clave: Eustoma grandiflorum, flor de corte, propagación, enraizamiento, auxinas.

\begin{abstract}
The growing interest in Lisianthus cultivation and the lack of available experimental data demands the study of the phenological and productive behavior of this species. One of the main concerns of the flower producer is the agronomic management and obtaining plants of this crop. The objective of this study was to evaluate the propagation technique and its effect on rooting of cuttings with different auxin concentrations. The plant material was extracted from donor plants which were thinned and topped to make them more vigorous. The first experiment used a closed system (CS) covered with transparent polyethylene, irrigating the substrate to saturation. The second experiment used a table with fogging system, with spray nozzles for irrigation. The design was randomized complete blocks; the Duncan test was used to analyze differences between means. The treatments were different concentrations of indole butyric acid (IBA): T0, control without IBA; T1, $250 \mathrm{ppm} \mathrm{IBA;} \mathrm{T2,} 500 \mathrm{ppm} \mathrm{IBA} \mathrm{and} \mathrm{T3,} 1000 \mathrm{ppm} \mathrm{IBA.}$ The results of the $C E$ experiment indicated that auxin application improved the rooting of cuttings, but there were no differences between treatments. In the TWF experiment the application of IBA improved the propagation; better results were produced by higher concentrations of the auxin.
\end{abstract}

Key words: Eustoma grandiflorum, cut flowers, propagation, rooting, auxins.

\section{Introducción}

El Lisianthus es una especie originaria de zonas áridas que se puede cultivar durante todo el año sin necesidad de estructuras costosas que pueden salir al mercado en contraestación, obteniendo mejores precios. La propagación del Lisianthus se realiza por semillas, aunque Melgares (1996) señala que existe la posibilidad de su multiplicación mediante esquejes o tejido in vitro. Maldonado y Contreras (2005) indican que el tiempo de germinación y crecimiento de la radícula se extiende por 10 a 14 días y, desde el trasplante hasta la obtención de los plantines varía de 10 a 19 semanas, según la época

\footnotetext{
1 Departamento Producción Agrícola, Universidad de Tarapacá, Chile.

* Autor por correspondencia: esotomay@uta.cl, conesotomayor@hotmail.com
}

Fecha de Recepción: 14 Junio, 2016.

Fecha de Aceptación: 30 Septienbre, 2016. 
de siembra, completando así entre 70 a 126 días. Verdugo (1994) indica que los esquejes tardan más de dos semanas en enraizar una vez trasplantado y que su sobrevivencia es baja. Melgares (1997) agrega que el ciclo de las variedades de macetas es muy corto, oscila entre ocho a diez semanas (55 a 70 días), desde la plantación de las plántulas en la maceta hasta su venta. Sotomayor et al. (2011) han conseguido enraizar en macetas de turba un cien por ciento esquejes de Lisianthus utilizando ácido $\beta$-indolbutírico. Como una manera de mejorar el tiempo de propagación se ha realizado este trabajo cuyo objetivo fue establecer dos experimentos para evaluar la técnica de propagación y su efecto en el enraizamiento de esquejes bajo distintas concentraciones de auxinas

\section{Materiales y Métodos}

El trabajo se realizó en la Parcela Experimental de la Universidad de Tarapacá, ubicado en el km 12 del valle de Azapa a $250 \mathrm{msnm}$. El cultivar utilizado fue cv. Abc 2-3 Blue Rim. Los experimentos se establecieron en un invernadero con cubierta de policarbonato con sistemas tecnificados para el control de la temperatura ambiental y humedad relativa. Las mesas de propagación contaban con termostatos para controlar la temperatura del sustrato, en un rango de 24 a $26^{\circ} \mathrm{C}$, según lo indicado por Sotomayor (1999). Se utilizó perlita tipo B12, descrita por Abad (2004) como sustrato en las mesas de propagación. Sobre esta se colocaron los contenedores de plástico de 5 x 6 x $3 \mathrm{~cm}$ basal, con capacidad para 50 esquejes. El sustrato de cada contenedor fue una mezcla de turba $(67 \%)$ y perlita (33\%).

Se realizaron dos experimentos. En el experimento en mesa cerrada (MC) se colocó una cubierta de polietileno transparente de $0,13 \mathrm{~mm}$ de espesor, manteniendo una humedad relativa entre 80 a $90 \%$. Sobre la perlita se colocaron los contenedores y se procedió a regar el sustrato hasta su saturación. Semanalmente se aplicó un riego por aspersión para mantener la humedad del sistema Sotomayor (1999). En el experimento de mesa bajo nebulización (MBN) se utilizaron boquillas atomizadoras, ubicadas cada $35 \mathrm{~cm}$ por encima de las hojas. El riego se programó con una duración de 12 segundos cada 20 minutos desde las 08:00 hasta las 19:00 horas.

El material vegetal fue extraído de plantas madre una vez realizado el raleo de tallos y pinzamiento. A cada esqueje o estaquilla se le dejaron cuatro nudos y dos hojas en la parte superior, procediendo el corte por encima del nudo apical, se les dejó dos nudos libres de hojas en la parte basal, haciendo el corte debajo del nudo (Figura 1). Se aplicó Previcur N (20 $\mathrm{cm}^{3} \mathrm{~L}^{-1}$ ) para prevenir enfermedades (Sotomayor et al., 1999). Los esquejes fueron tratados con auxina en forma de ácido $\beta$-indolbutírico (AIB). Las estaquillas fueron sometidas a una inmersión basal con una solución hidroalcohólica al 50\% con concentraciones de 0, 250, 500 y 1.000 ppm de AIB, durante 5 segundos. Los tratamientos fueron: Testigo (T0), sin AIB; 250 ppm de AIB (T1); 500 ppm de AIB (T2) y 1.000 ppm de AIB. A los 60 días se hizo el recuento del porcentaje de enraizamiento en cada uno de los tratamientos. El diseño experimental fue de Bloques Completamente al Azar y 30 repeticiones. Para el análisis de varianza y la separación de medias se usó el test de Duncan $(\mathrm{p} \leq 0,05)$.

\section{Discusión de Resultados}

En la Tabla 1 se observan los resultados del porcentaje de enraizamiento de los esquejes de Lisianthus en ambos experimentos, según tratamiento. En ambos experimentos se observa una mayor rizogénesis al someter las estaquillas a la acción de una auxina, coincidiendo con los resultados de Winarto et al., 2015.

En el caso del Experimento MC, se observa que hay diferencia significativa entre el testigo y la aplicación de auxina, independientemente de la concentración de ácido $\beta$-indolbutírico a las que fueron sumergidos los esquejes. Estos resultados sugieren que se pueden enraizar los esquejes de Lisianthus con una dosis de 250 ppm cuando se usa la técnica de mesa cerrada para la propagación de las estaquillas.

En el experimento con mesa bajo nebulización, se observa que hubo diferencia significativa al someter los esquejes a la acción de AIB. Esta diferencia se observa también en la concentración de la auxina, lográndose un $100 \%$ de enraizamiento cuando las estaquillas fueron sumergidas a la mayor concentración. Los resultados sugieren que, bajo la técnica de mesa bajo nebulización, hay una relación directamente proporcional entre la concentración de auxina y el porcentaje de enraizamiento.

Al observar el testigo en ambos experimentos, se observa que en mesa cerrada el porcentaje de enraizamiento es menor al 50\%. En la mesa bajo nebulización el porcentaje aumenta al $60 \%$. 

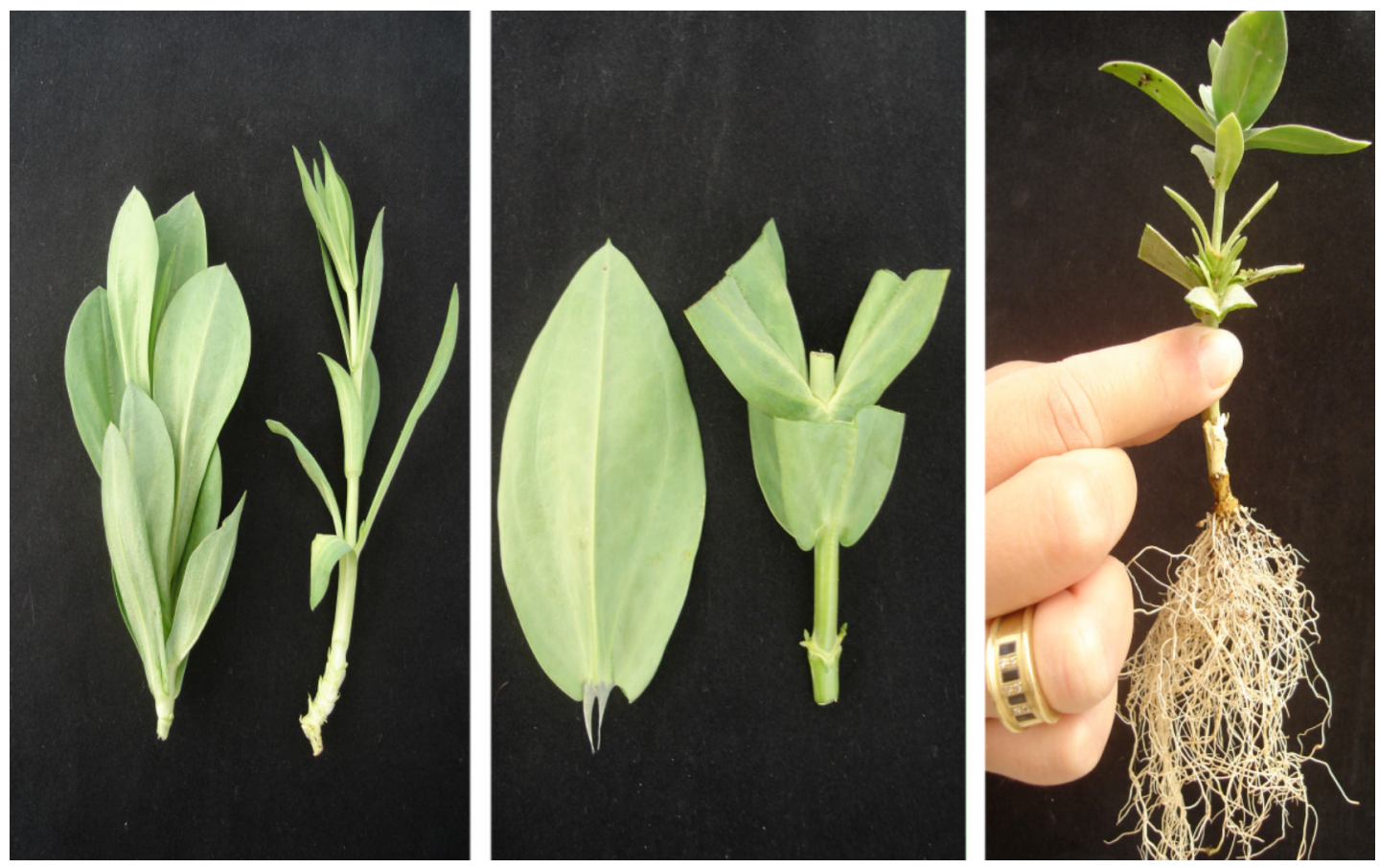

Figura 1. Deshoje de esquejes o estaquillas (izquierda); Poda de hojas en estaquillas (centro); Planta de Lisianthus enraizada (derecha).

Tabla 1. Porcentaje de enraizamiento de Lisianthus por Experimento en Mesa Cerrada (MC) y Mesa Bajo Nebulización (MBN), según concentración de ácido $\beta$-indolbutírico (AIB): $\sin$ AIB (T0), 250 ppm AIB (T1), 500 ppm AIB (T2) y 1.000 ppm AIB (T3).

\begin{tabular}{ccc}
\hline & Experimento MC & Experimento MBN \\
\hline T0 & $48 \mathrm{~b}$ & $60 \mathrm{c}$ \\
T1 & $87 \mathrm{a}$ & $75 \mathrm{~b}$ \\
T2 & $93 \mathrm{a}$ & $92 \mathrm{~b}$ \\
T3 & $80 \mathrm{a}$ & $100 \mathrm{~b}$ \\
\hline
\end{tabular}

Test de Duncan $\mathrm{p} \leq 0,05$. Letras iguales indican que no hay diferencia significativa.

\section{Conclusiones}

Con los resultados de este trabajo se puede concluir que los esquejes de Lisianthus responden positivamente a la aplicación de auxina para la rizogénesis. En mesa cerrada se recomienda una dosis de $250 \mathrm{ppm}$ de AIB. En mesa bajo nebulización se obtiene un $100 \%$ de enraizamiento al aplicar $1.000 \mathrm{ppm}$ de AIB y no hay diferencia significativa entre dosis de $250 \mathrm{ppm}$ y $500 \mathrm{ppm}$ de ácido $\beta$-indolbutírico.

\section{Literatura Citada}

Abad, M.; Noguera, P.; Carrión, C.

2004. Los sustratos en los cultivos sin suelos. En: Tratado de cultivo sin suelo. Urrestarazu, M (ED). Mundi-Prensa, Madrid, pp. 113-158.

Maldonado, P.; Contreras, J.

2005. Lisianthus, producción de plántulas. Tierra Adentro, 60: $39-41$.

Melgares, J.

1996. El Cultivo de Lisianthus (I Parte) Horticultura. Revista de Hortalizas, Flores, Plantas, Ornamentales y Vivero, 15 (4): $13-16$

Melgares, J.

1997. El Cultivo de Lisianthus (II Parte) Horticultura. Revista de Hortalizas, Flores, Plantas, Ornamentales y Vivero, 15 (5): 47-50.
Sotomayor, E.; Escobar, M.; Rosas, C.

2011. Propagación del Lisianthus cv. Azul por esquejes en macetas de turba bajo nebulización, con distintas concentraciones de ácido $\beta$-indolbutírico. Idesia, 29 (1): 99-102.

Sotomayor, E.

1999. Propagación del olivo Olea europaea L. cv. Azapa por estaquillado semileñoso bajo diferentes medios de enraizamiento. Idesia 17 (1): 9-17.

Winarto, B.; Rachmawati, F; Setyawati, A.; Da Silva, J.

2015. Leaf-derived organogenesis in vitro for mass propagation of Lisianthus (Eustoma grandiflorum (Raf.) Shinn.) Emir. J. Food Agric., 27 (6): 495-501. 
